(C)2009 IEEE. Personal use of this material is permitted. However, permission to reprint/republish this material for advertising or promotional purposes or for creating new collective works for resale or redistribution to servers or lists, or to reuse any copyrighted component of this work in other works must be obtained from the IEEE. 


\title{
Applications of Wireless Sensor Networks in Pharmaceutical Industry
}

\author{
Manohar Potdar ${ }^{1}$ Atif Sharif ${ }^{2}$ Vidyasagar Potdar $^{2}$, Elizabeth Chang ${ }^{2}$ \\ ${ }^{1}$ Department of Pharmacy, Bharati Vidyapeeth University, Pune, India \\ ${ }^{2}$ Digital Ecosystems and Business Intelligence Institute, \\ Curtin University of Technology, Perth, Western Australia \\ potdar_manohar@yahoo.com.au, atif.sharif@postgrad.curtin.edu.au \\ v.potdar@curtin.edu.au, e.chang@curtin.edu.au
}

\begin{abstract}
Pharmaceutical and healthcare industries are highly regulated industries in each and every nation around the globe. Every nation has a governing body like USFDA, TGA, and M.H.R.A, Schedule-M etc. that assures that the medicines are manufactured under strict guidelines to ensure highest quality of manufactured products. However assuring high quality products requires that the manufacturing facility is monitored $24 \times 7$ and this monitoring requires the use of high end networking technology. Currently pharmaceutical plants capture this information using a number of scalar sensors that measure temperature, pressure, humidity etc. In some developing nations this sensed information is manually recorded by a supervisor in charge. This often results in data entry errors that need to be addressed. This paper aims at identifying current technologies that can be used by Indian Pharmaceutical Industries to capture this information and process it in real time to provide the kind of track and trace which so far is not implemented in majority of Indian pharmaceutical companies. We specifically focus on identifying the importance of Wireless Sensor Networks and how it can be implemented in pharmaceutical manufacturing plants.
\end{abstract}

\section{Introduction}

The realization of next generation ubiquitous and pervasive healthcare systems will be a challenging task, as these systems are likely to involve a complex structure. Such systems will consist of various devices, ranging from resource constrained sensors and actuators to complex multimedia devices, supporting time critical applications. Medicine production and medical diagnostics are two industries where precision and reliability are a must. Meeting safety requirements and complying with federal regulations are a large part of the task. Now a day's pharmaceutical industry aimed to produce quality production in a cost effective manner. In order to achieve this objective the production facility must be monitored for any uncertain change like change in pressure, temperature or humidity etc which are quite vital elements to be controlled. The produced stock must be saved in storage rooms. And again for medicinal storage strict conditions of temperature, humidity etc may apply. Old practice of manual recording of environmental variables (like temperature, pressure, humidity etc) based pharmaceutical facility control may leads to errors and require dedicated staff to look after the pharmaceutical facility.

Another key point which attracted world's attention is the transportation of the medicines to various parts of country or world. As there are few well renowned pharmaceutical manufacturers which are producing life saving drugs and shipment of these drugs do require special attention. Long Distance transportation of temperature and humidity sensitive medicines are required to be transported at specified conditions (e.g. Temp of 2 to $8 \mathrm{C} \&$ humidity of less than $50 \%$ ). In such a cases one or more (say 4 to 5) temperature, Humidity sensing \& recording devices are sent along with the shipment so that the conditions during transportation can be recoded \& verified at the destination. This is required as per T.G.A. Australia regulation and also with USFDA. In USFDA such regulation is specified in 21CFR part 211 \& monitoring must comply with 21CFR Part 11(Part 11 deals with Calibration \& Validation Computer \& electronic Systems).

The rest of the paper is structured as follows. Section 2 lists and explains the information that is required to be captured in a pharmaceutical manufacturing plant to comply with local health authorities like USFDA, TGA, FDA etc. This section will outline how this information is captured currently and lists some issues with the current approaches. Section 3 gives an introduction to Wireless Sensor Network technology and how it can address some of the issues outlined in section 2. Section 4 then studies the current commercial available WSN technology solutions in the pharmaceutical and health arena and analyses these solutions on its practicality. And finally Section 5 concludes the paper with future research directions.

\section{Information Requirements}

The Pharmaceutical industry in India is regulated by the Drugs \& Cosmetics Act 1945 under the 
control of Foods \& Drugs Administration of India. The Schedule-M of the drugs \& cosmetics act specifies the regulation related to "Good Manufacturing Practice" (GMP).

The important guideline of GMP is that the environment, where the medicines are produced must meet the specific requirements for temp, humidity etc, similarly the process used in pharmaceutical manufacturing also require strict control on the process parameter like pressure, temp, humidity etc. The regulation also requires the condition maintained during the processing and should be made available during FDA inspections to the inspecting authorities.

Such records can be maintained as soft copies but when demanded by FDA authorities, it should be provided as hard copies for inspection purpose. The electronic recording devices must be 21CFR Part 11 compliant (21CFR part 11 is a regulation which controls the use of computer \& similar electronic devices in pharmaceutical manufacturing).

We now list the two main areas within pharmaceutical manufacturing where control information needs to be captured on a regular basis. This includes:

1. Environmental Control, and

2. Process Control

\subsection{Environmental Control}

Within the realm of environmental control the key parameter that needs to measured on a regular basis are: room temperature, percentage relative humidity and differential pressure.

\subsubsection{Room temp and percentage relative humidity}

In pharmaceutical plants all the process and store rooms need to maintain specific temperature and humidity to assure the quality control standards set by the regulatory bodies as pharmaceutical products are highly sensitive to temp \& humidity. The optimum temperature should generally be between $20^{\circ} \mathrm{C}$ to $25^{\circ} \mathrm{C}$ and percentage relative humidity should be in between $40 \%$ to $50 \%$. This however will change as per the manufacturing specifications of the products and the pharmaceutical manufacturer has to prove that the conditions of temperature \& humidity were well within the specifications when the pharmaceutical products were manufactured. This has to be done through recorded evidence.

\subsubsection{Differential pressure in the room}

Pharmaceutical manufacturing processes are classified as critical \& relatively less critical. Critical processes are those where extreme control on the purity of air is required. (The air quality is classified as grade A, B,C \& D by M.H.R.A (U.K.) Guide line). This is achieved by filtering the air through suitable filter called HEPA (high efficiency particulate air). The more critical operation, e.g. filling sterile liquid or powder in injectables are carried out in relatively high pressure room (about $1.5 \mathrm{ml}$ of water gauge in the adjacent rooms). This helps in maintaining air quality in critical rooms since when door is opened between a critical and less critical room, then better quality air flows from critical to less critical room. Hence the data of differential pressure (that is difference of pressure of air in two or more adjutant room) is required to be captured, recorded and maintained for future reference and use.

These data are presently recorded either manually or by a Building Management System (BMS). This is an area where WSN can play a very important role by room wise sensing, capturing, recording, and maintaining the data on temperature, humidity and air pressure on $24 \times 7$ basis, which is one of the major requirement of F.D.A. auditors through out the world.

\subsection{Process Control}

Pharmaceutical manufacturing processes require very strict control on their parameters. The actual observations of the parameters are to be recorded \& maintained during the process. Following are the key processes \& their parameters which are required to be captured \& recorded continuously to adhere to the regulatory requirements.

\subsubsection{Tablet Granulation Process}

During the tablet granulation process the following parameters are required to be measured:

1. Temperature of air in fluidized bed drier.

2. Velocity of air in humidity of the air.

3. Change in $\%$ humidity of the air entering \& exiting from fluidized bed drier.

4. Electric current drawn by rapid mixer granulator during granulation process, since the end point of granulation is detected by sudden change in current drawn.

\subsubsection{Tablet Compression Process}

During the tablet compression process the speed of rotation of machine is required to be measured, since the equipment is validated for a specified range of speed of rotation.

\subsubsection{Sterilization of Injectable Containers using} Tunnel Sterilizer

The key parameters measured during this process include:

1. Temperature of Sterilization

2. Time of sterilization

3. Speed of conveyer belt of the sterilizing tunnel.

\subsubsection{Sterilization of Materials using Autoclave}


The key parameters measured during this process include:

1. Temperature of the chamber of the autoclave.

2. Pressure of steam in the chamber of the autoclave.

\subsection{Other areas}

There are some other areas where monitoring is required like monitoring the health of mice (or mouse), which is often used in testing the drug.

If all these monitoring activities could be automated it will provide a platform where the right information will be available at the right time and in the right format for taking informed decisions. This automation can be achieved by implementing a wireless sensor network infrastructure within a pharmaceutical plant. WSN is a cutting edge technology and its applications are just limited by imagination.

Before discussing the main application of WSN for the pharmaceutical industry we would like to introduce the basics of wireless sensor networks in the next section, to give the reader an understanding of the basic components within this technology and appreciate its importance for the pharmaceutical sector.

\section{Wireless Sensor Networks}

WSN is composed of intelligent embedded nodes (or motes) capable of communicating over wireless link with base station and other nodes. Researchers are working on WSN for environment monitoring $[1,2]$, robotics and for security check and balance applications. The system comprising WSN network and Radio Frequency Identification (RFID) tags is shown in Fig. 1. The network is comprised of

1. Server/ Base station

2. Sink Node

3. WSN nodes

4. RFID tag's

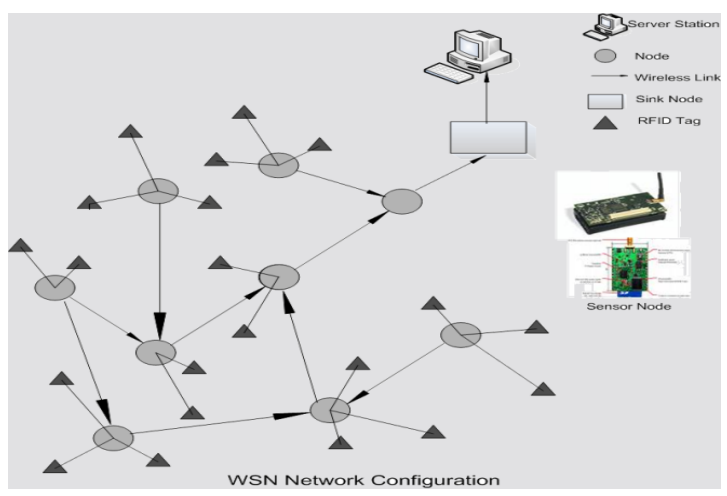

Fig. 1 RFID-WSN Network Configuration

Server/Base stations are treated as an endpoint of the network, which gathers data coming from multiple nodes, processes it and keeps record of it.
The nodes does not directly feedback to the server, infact the data from all nodes come to the sink node first (over wireless link) and then from the sink node it is passed to the server either over the wireless channel or by the use of wired/optical medium. The server has to keep record of changes so its storage capacity and computational capability is high enough to meet the application demands.

Fig. 2 describes the functional components of WSN/Sink node. The Sink node and the usual WSN node are same by functionality but they only differ with respect to their computational power, storage capacity, data rate etc. Generally sink node has high computational capability, large storage capacity and fast data rate support (since it gathers data from all the nodes). The generic WSN node comprises of the following
1. Sensor Unit
2. Central Processing Unit
3. Communication Unit
4. RFID Tag
5. Power

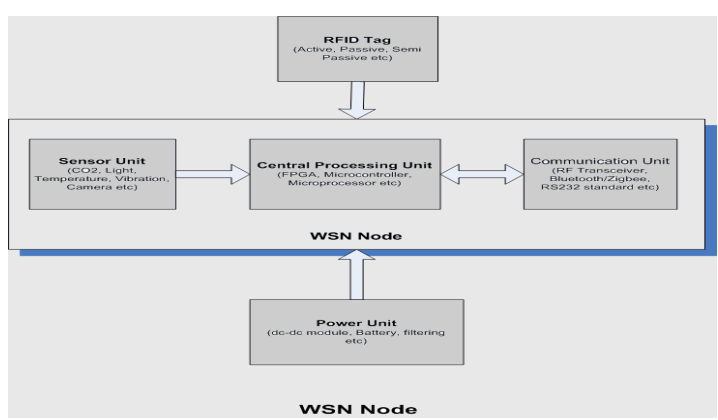

Fig. 2 WSN Node Architecture

The sensor unit is comprised of a number of sensors for measuring temperature, humidity, light intensity, gas measurement, vibrations and CMOS camera for video monitoring. The sensors convert the measured environmental changes into electrical signals for further processing by the central control unit.

CPU is considered as the heart of WSN node (best describes the node's computational power and its energy consumption hub) and is responsible for gathering data from group of sensors and to process it to some useful compact information prior to the transmission. Microcontroller, Microprocessor or an FPGA/CPLD may be used as CPU depending upon the complexity of the node. For lesser complexity microcontrollers are generally used but for more complex applications involving video or complex sensor network data microprocessor or an FPGA would be an ideal choice.

The communication unit interacts with the CPU to send data over the RF link to the neighbouring node or to the sink node. Various radio standards semiconductor chips exits for data transmission like TR1000, CC1000, CC2420/31, 
Bluetooth/Zigbee and UWB having variable data rate and power consumption. Additionally if RFID is used then this unit may also have RFID reader support so as to interact with the RFID tags.

Power unit mostly comprised of AA size batteries and DC-DC module for feeding the WSN node. While designing WSN, network designers should consider energy consumption of node, which should be kept to minimal for the maximal life time of the network. Various battery technologies exist like Lithium Manganese Oxide $\left(\mathrm{LiMnO}_{2}\right)$, Nickel Cadmium (NiCd), Nickel Metal Hydride (NiMH), Lithium ion (Li-ion), Lithium polymer (Li-polymer) etc and choice is entirely based on the nature of the application.

RFID tags (active, passive or semi-passive) are generally used to provide object information and to track things. Using WSN, RFID's addressable range can be exponentially increased. However using RFID, security [4] is a big concern and to counter this various security and authentication protocols exists, the choice of which entirely depends up on the node's computational resources, power source and their application.

\section{Applications of WSN in Pharmaceutical Industry}

WSN technology has made significant inroads within the pharmaceutical and healthcare industry in addressing some of the key problems like monitoring the status of pharmaceutical plants, and patients, preventing drug counterfeiting, monitoring the health of mouse etc.

\subsection{Anti-Counterfeit}

RF chips have already been used in a wide variety of applications in many different types of organizations including tamper detection and counterfeiting. In the pharmaceutical industry, IBM is using RFID and sensor technology to help manufacturers and distributors protect consumers from counterfeit drugs [3].

\subsection{Facility Monitoring System}

WSNs have gained a key importance in monitoring the pharmaceutical facility [8]. Knowing this importance of WSNS Adaptive solution in collaboration with Facility Monitoring Solutions Ltd has successfully installed WSNs for monitoring application. In London NHS hospital WSNs has been deployed for facility monitoring including multi-area pharmacy clean room, packaging area and stores. The deployed network monitors the scalar parameters like ambient temperature, humidity and refrigeration temperature. The data from the entire Network is used by the FMS monitoring software for various control settings.

\subsection{Mice Monitoring System}

In pharmaceutical and biotechnology research labs mice is generally used as a test model. Researchers practice various newly researched medicinal effects on mice while observing their behaviour. The disturbance caused by the human presence may affect the experimental results greatly. To overcome this problem the WSNs plays a vital role for remotely accessing the experimental data. For this special purpose a mote's hardware has been designed that fulfil the application's requirements. The observed data, after pre-processing, is transmitted by nodal network to the gateway from where it is being sent to the control station for display. The system is robust enough and withstand against possible node failure. The SQL based data stations are used for storing the time stamped processed experimental data. And this data reading are collected after every fifteen minutes and is quite helpful for bringing improvement in the research.

\subsection{Magneto Inductive Sensors to detect Passage of Pills}

Wireless Sensors [6] are also helping physicians in getting the patients record of taking the medications appropriately and timely. Magnetic sensor based system using an array of magnetoinductive sensors is used for this type of monitoring application. Such sensors are mounted around the patient's neck to detect the passage of pill which is embedded with small permanent magnet. During the passage of pill the sensor mounted around the patients neck detects it and send the time tamped data to software running on PDA for possible retrieval by the physician. The initial design was based on the off-the shelf components and customized PCB design. The proposed system showed good results having detection accuracy of $94.4 \%$.

\subsection{Pharmaceutical Facilities Monitoring using Sensicast Systems}

WSNs greatly used in pharmaceutical test laboratories, manufacturing area and storage locations to report any change in the defined control parameters set by the ambient temperature, humidity conditions etc. Another break through is the development of Sensicast Wireless Network which gathers information from the monitoring locations, storage, test lab, manufacturing area and shipping point etc, using the smart embedded module which intern transmit wirelessly to company's network infrastructure. The data reliability and redundancy are the key parameters which are met by this network configuration. The gateway gathers the sensed information from the sensory network and displays it for possible alarming conditions or for facilities' internal automation, process and control systems. 
SensiNet [9] is embedded with self intelligence and thus prevents human involvement. It helps in managing the production facility to follow the FDA regulation and keep the collected data well documented.

\subsection{Cold Chain Management using WSN}

For Pharmaceutical manufacturers it is important to have a controlled supply chain temperature (normally 2 to 8 degree Celsius) during medicine production. Cold chain [12] can efficiently manage the pharmaceutical supply chain there by reducing the risk and cost. RFID and WSN, key elements of pervasive computing, are being used in the modern cold chain for monitoring the temperature sensitive products. Such a system is also being used in monitoring the inventory and transportation of such temperature sensitive products. In this case RFID is used for track and trace while WSNs is being used for conditional monitoring. The integration of the two pervasive elements has significantly enhances the existing track and trace applications. Thus the availability of product's trace history and conditional record helps in various key decision processes [11]. The product should be monitored for its entire life from the manufacturing point till the final destination. This is basically the key objective of cold chain. During product shipment the RFID tag (for track and trace) and WSN node (embedded with scalar sensors for condition monitoring) are mounted to the product pack. This helps in real time monitoring of the product. The system is scalable by nature and gathers information from the nearby RFID sensory nodes. The gathered information is then transmitted wirelessly to the sink node which in turn connected to some computer or internet from where the user gathers information for analysis.

Although most of the available systems that incorporates RFID or WSN or both are basically researched to monitor the pharmaceutical infrastructure and environmental monitoring for drug storage and transportation etc. The research may be carried out in securing the communication from external threats. Another dimension in which research may be invited is to incorporate the multitiering approach for pharmaceutical infrastructure monitoring involving visionary sensors besides the use of scalar sensors for measuring the temperature, humidity, pressure etc. Also reliability of the data transfer especially the alarming conditions are of great importance. This thing will open a new vision for the reliable communication protocol development.

\section{Conclusion \& Future Work}

In this paper we studied the existing WSN technology that can be used in pharmaceutical \& health care areas. The current state of the art technologies were analysed based upon how well they can meet the information requirements laid by the regulatory authorities. The next stage of this collaborative research would look at the open research directions within these current solutions and aim to develop next generation WSN techniques specifically for the pharmaceutical industry.

\section{References}

[1] A. M. BRATKOVSKI, "Monitoring an environment using RFID assembly." vol. WO/2006/094085, W. I. P. Organization, Ed. USA: HEWLETT-PACKARD Development Company, L. P., 2006, p. 13

[2] G. BUNZA, J.HUDNUT, Steven, W., "Environmental condition detecting system using geospatial images and associated methods" in WO/2008/088430, W. I. P. Organization, Ed. USA: VIGILAN, INCORPORATED 24.07.2008.

[3] Andrew K. Burger, RFID and Wireless Sensor Networks in the Supply Chain, http://www.technewsworld.com/story/wireless/554 92.html, 2007, 02 ${ }^{\text {nd }}$ Dec, 2008.

[4] Sharif, A. Potdar, V, "A Critical Analysis of RFID Security Protocols," in 22nd International Conference on Advanced Information Networking and Applications - Workshops, 2008. AINAW 2008. ,2008, pp. 1357-1362.

[5] "Emerson uses Dust Networks" ${ }^{\mathrm{TM}}$ WSN products in new Smart Wireless solutions for mainstream manufacturing", http://www.emersonprocess.com/home/news/pr/610 _dust.html, $03^{\text {rd }}$ Dec, 08

[6] M. Xueliang Huo Ghovanloo, "A Wireless Pharmaceutical Compliance Monitoring System Based on Magneto-Inductive Sensors," Sensors Journal, IEEE, vol. 7, pp. 1711-1719, Dec. 2007.

[7] B.J. Y. Young-I Kim, Jae-Ju Song, Jin-Ho Shin, and Jung-I Lee "Implementing a Prototype System for Power Facility Management using RFID/WSN", in Proceeding of world academy of science, engineering and technology, Venice, Italy, Nov. 2006, pp. 1- 6.

[8] Sjbaker, "Wireless sensor network plays key role in London Hospital pharmacy compliance monitoring system", http://www.adaptivewireless.co.uk/?cat=6, 2007, $29^{\text {th }}$ Nov, 2008

[9] "Sensicast Ships WSN for Pharma, Life Sciences",

http://process.sensorsmag.com/sensorsprocess/artic le/articleDetail.jsp?id=421982, 2007 
[10] RFM Wireless Sensor Networking Products: http://www.rfm.com/news/nr_xdm2140.shtml，27 $7^{\text {th }}$ Nov, 2008

[11] J. Mitsugi et al., "Architecture Development for Sensor Integration in the EPCglobal Network", AutoID Labs White Paper WP-SWNET-018, July 2007

[12] W. C. Fu, Y S Aung, M M Makatsoris, C Oh, C H, "WSN based intelligent cold chain management," in The 6th International Conference on Manufacturing Research (ICMR08), UK, September, 2008, pp. 353-360. 